\title{
Doppler Ultrasound in Prostate Cancer: It's Utility for the Diagnosis of High-Grade Tumors
}

\begin{abstract}
Marcos Álvares de Campos ${ }^{1,2}$, Lucas Mendonça Darwich ${ }^{1,2}$, André Thomaz Ezequiel Pereira ${ }^{1,2}$, Viviane Fernandes de Castro Rodrigues ${ }^{1,3}$, Renato Alves Carvalho $^{1,3}$, Elisa Carvalho de Siqueira ${ }^{1 *}$, Fabrício Tinoco Alvim de Souza ${ }^{1}$, Fabiano Franco Monteiro Prado ${ }^{1,3}$, Tiago Paes Gomide ${ }^{1,3}$, Paulo Ramos Botelho Antunes ${ }^{1,3}$, Maria Carolina Barbosa Álvares ${ }^{1,3}$ and Luiz Felipe de Campos-Lobato ${ }^{2,4}$
\end{abstract}

${ }^{1}$ Research Group on Diagnostic and Therapeutic Radiology, Santa Casa Hospital, Brazil

${ }^{2}$ Faculty of Health and Human Ecology (FASEH), Brazil

${ }^{3}$ Service of Radiology, Santa Casa Hospital, Brazil

${ }^{4}$ School of Medicine, Federal University, Brazil

*Corresponding author: Elisa Carvalho de Siqueira, Research Group on Diagnostic and Therapeutic Radiology, Avenida Francisco Sales, 1111, Bairro Santa Efigênia, 30150-221 Belo Horizonte, Minas Gerais, Brazil, Email: elisadesiqueira@hotmail.com

\section{Abstract}

Purpose: The aim of the present study was to evaluate the association between prostate vascularization seen in Doppler ultrasound and histopathological grade (Gleason score) in patients with a diagnosis of prostate cancer.

Methods: A Gleason score $>7$ was the dependent variable and Doppler ultrasound findings (vascular analysis, presence of nodule and prostate weight) were the independent variables. Univariate analysis was performed considering advanced tumors (Gleason >7) as the dependent variable and area of hypervascularization, age and PSA as the independent variables. Multivariate analysis was performed using a binary regression model with the occurrence of advanced tumors (Gleason $>7$ ) as the dependent variable.

Results: In the univariate analysis, samples with Gleason $\leq 7$ had a lower chance of being hypervascularized (OR: $0.44,95 \%$ CI: 0.29-0.69), whereas those with Gleason scores $>7$ had a fourfold greater chance of being hypervascularized (OR: 4.136, 95\% CI: 2.598-6.554, p<0.001). Moreover, hypervascularized tumors had a 7.4-fold greater chance of having a score $>7$.

Conclusion: The present study reveals an association between tumor hypervascularization detected using Doppler ultrasound and higher Gleason scores (more aggressive tumors), enabling an indirect inference of a worse prognosis for hypervascularized prostatic tumors. These findings should be confirmed in longitudinal studies.

Keywords: Prostate cancer; Gleason Hypervascularization; Doppler; Prostate carcinoma; Ultrasonography; Biopsy

Abbreviations: PSA: Postate Secific Antigen; MRI: Multiparametric Magnetic Resonance Imaging; ISUP: International Society of Urological Pathology; TNM: Tumor Node Metastasis; TRUS: Transrectal Ultrasound.

\section{Introduction}

Prostate cancer is the most common noncutaneous cancer among males [1]. Most cases are found in patients 


\section{Clinical Pathology \& Research Journal}

older than 75 years of age, with only $1 \%$ of cases found in men younger than 50 years of age [2]. The main methods for investigating and detecting prostate cancer are a digital rectal exam, the serum concentration of prostate specific antigen (PSA) and transrectal ultrasound biopsy followed by histopathological analysis [3]. Among the diagnostic tools used today to date for prostate cancer, multiparametric magnetic resonance imaging (mpMRI) has demonstrated an improvement in detection and characterization of prostate cancer [4]. Although well validated in terms of diagnostic accuracy, mpMRI requires technical rigour, patient's tolerability and safety, expertise in interpretation, and high costs [5]. Conventional imaging techniques, as conventional ultrasound, that are optimized for well-defined, round tumor masses may be less effective for the prostate considering that $85 \%$ of prostate cancer cases is multifocal [6]. Due to the varied prognosis, knowledge of prognostic markers is useful for better counseling and the definition of the course to be taken. Since there is a correlation between early detection of tumors and improved prognosis, enhancing current tumor imaging approaches is critical.

The increased of vessel density has a predictive value in the identification of high-risk patients with a poor prognosis [7]. Considering that have been demonstrated the role of angiogenesis in solid neoplasia which correlates with rapid tumor growth and potential for metastasis [8] ultrasound Doppler techniques can be used. This technique offers a low-cost and noninvasive approach with which to measure changes in vascular and bloody features [9].

Studies describe the prognostic importance of angiogenic factors in different types of cancer (lung, ovarian, stomach cancer and squamous cell carcinoma of the head and neck) [10-13]. As with other tumors, an angiogenesis is found in prostate cancer [14-16] and this characteristic can be evaluated using Doppler, although the efficiency of this technique is debated [16]. Although Doppler techniques may provide some improvement in the imaging detection of prostate cancer, targeted biopsy based on conventional ultrasound with Doppler is not sufficient to replace systematic biopsy [17]. Gleason grading system is a histopathological grade in diagnosis of prostatic neoplasm [18]. Khanduri, et al. Reported that transrectal ultrasound with color Doppler flowmetry can play an important role in the detection of prostate cancer, with high sensitivity as well as specificity, comparing with histopathological data [18]. Up to now, it is worth mentioning that the most important prognostic parameters to consider are the PSA level, the International Society of Urological Pathology (ISUP) graduation and the Tumor, Node, Metastasis (TNM) staging system [19].

Based on this context, the aim of the present study was to evaluate the association between prostate vascularization, with color Doppler fluxometry and histopathological grade (Gleason score) in patients with a diagnosis of prostate cancer.

\section{Methods}

\section{Sample}

The retrospective cross-sectional observational study received approval from the Human Research Ethics Committee of our service. The study involved 188 patients with prostate cancer confirmed by anatomopathological analysis that underwent a Doppler ultrasound exam by a single specialist with more than 20 years of experience with this type of exam. It was conducted at the Radiology service of the authors' Hospital. Patient age and serum prostatespecific antigen (PSA) level were also recorded.

\section{Ultrasound Analysis}

All patients who fulfilled the inclusion criteria were examined by trans rectal ultrasound (TRUS) with a color Doppler for the detection of prostatic lesion using Toshiba Xario Istyle (Toshiba Corporation, Tokyo, Japan) ultrasonography color Doppler machine, with a TRUS probe (6 MHz). After the analysis of the exams, the sample was divided into two groups based on the presence or absence of hypervascularization areas (dominant increase in the number of vessels) in prostate (Figure 1).

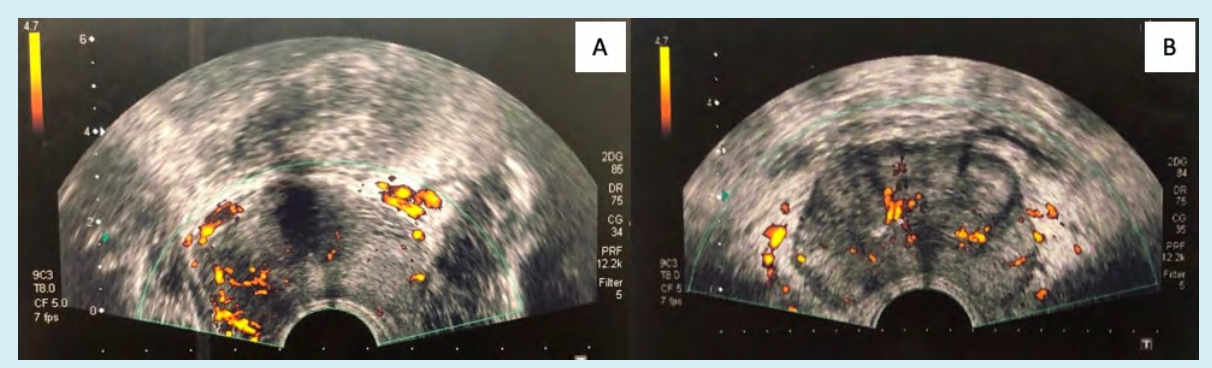

Figure 1: Representative images of doppler ultrasound findings. (A) Hyper vascularized area in peripheral prostatic zone. (B) Area with normal vascularization in peripheral prostatic zone. 


\section{Clinical Pathology \& Research Journal}

Hypervascularization was defined as the presence of a high color code found using power Doppler ultrasound [20] which was based on the association between the increase in the color code and an increase in blood flow.

\section{Histopathological Analysis}

The samples included in the present study were classified using the Gleason score described on the individual histopathological files. This grading system categorizes the two largest biopsy fragments on a scale of 1 to 5 based on the similarity of the neoplastic tissue to healthy tissue, according to International Society of Urological Pathology [21].

\section{Data Analysis}

The two groups were compared in terms of age, PSA, ultrasound findings and Gleason score. A regression model was created to evaluate the capacity of hypervascularization to predict tumor aggressivity. For such, the dependent variable was a Gleason score $>7$. The independent variables were the presence of a hypervascularized area and variables known to be associated with a worse prognosis: PSA $(\geq 20$ $\mathrm{ng} / \mathrm{ml})$ and $(>65$ y.o.) [22,23]. The categorical variables were described in absolute number and percentage values and compared using either the chi-squared test or Fisher's exact test. Continuous variables were expressed as median and interquartile range (IQR) and compared using the Wilcoxon test. For the evaluation of predictive factors of advanced tumors, the Gleason score was dichotomized as $\leq 7$ (low grade) and $>7$ (intermediate and high grade). This cutoff point was based on the definition of the American Cancer Society [24].

Univariate analysis was performed considering advanced tumors (Gleason $>7$ ) as the dependent variable and area of hypervascularization, age and PSA as the independent variables. Multivariate analysis was performed using a binary regression model with the occurrence of advanced tumors (Gleason $>7$ ) as the dependent variable. Covariates with a p-value $<0.10$ were incorporated into the multivariate model using a backward stepwise procedure. The final model included the most restricted subset of variables with statistical significance. Associations were expressed by odds ratios and respective $95 \%$ confidence intervals. The goodness of fit of the model was evaluated using the Hosmer-Lemeshow test. A p-value $\leq 0.05$ in the final model was considered indicative of statistical significance.

\section{Results}

188 patients were included in the study. All participants were treated at the radiology sector of our Hospital between February $5^{\text {th }}$ and December $17^{\text {th }}, 2018$. Median age was 67 y.o. (IQR: $62-72$ y.o.). Median prostate weight was $43.55 \mathrm{~g}$ (IQR: 33.63-59.88g). The Gleason score ranged from 6 to 10 (Table 1).

\begin{tabular}{|c|c|c|c|}
\hline Feature & $\begin{array}{c}\begin{array}{c}\text { Hypervascularization } \\
(n=34)\end{array} \\
\end{array}$ & $\begin{array}{c}\text { Normal vascularization } \\
(n=154)\end{array}$ & p-value \\
\hline Age (y.o.) ${ }^{1}$ & $67(61-72)$ & $67(63-73)$ & 0.314 \\
\hline Prostate weight (grams) ${ }^{1}$ & $52.55(38.50-96.60)$ & $41.15(33.00-58.30)$ & 0.013 \\
\hline Serum concentration of PSA $(\mathrm{ng} / \mathrm{ml})^{1}$ & $8.84(5.18-49.68)$ & $7.89(5.18-17.22)$ & 0.185 \\
\hline Presence of nodules ${ }^{2}$ & $11(17.74)$ & $51(82.26)$ & $<0.001$ \\
\hline Gleason $2^{2}$ & $0(0)$ & $0(0)$ & \multirow{8}{*}{$<0.001$} \\
\hline Gleason $3^{2}$ & $0(0)$ & $0(0)$ & \\
\hline Gleason $4^{2}$ & $0(0)$ & $0(0)$ & \\
\hline Gleason $5^{2}$ & $0(0)$ & $0(0)$ & \\
\hline Gleason $6^{2}$ & $9(20.93)$ & $34(79.07)$ & \\
\hline Gleason $8^{2}$ & $7(43.75)$ & $9(56.25)$ & \\
\hline Gleason $9^{2}$ & $13(48.15)$ & $14(51.85)$ & \\
\hline Gleason $10^{2}$ & $1(100)$ & $0(0)$ & \\
\hline Gleason $>7^{2}$ & $21(47.73)$ & $23(52.27)$ & \multirow{2}{*}{$<0.001$} \\
\hline Gleason $\leq 7^{2}$ & $13(9.03)$ & $131(90.97)$ & \\
\hline
\end{tabular}

Table 1: Characterization of the dichotomization sample (hypervascularization versus normal vascularization).

y.o.= years old.

${ }^{1}$ data were presented as median and interquartile interval

${ }^{2}$ data were expressed as absolute and percentage values.

PSA level ranged from 0.67 to $3332.60 \mathrm{ng} / \mathrm{ml}$. 


\section{Clinical Pathology \& Research Journal}

A total of $33.88 \%$ of the samples had prostatic tumors with nodules and $66.12 \%$ did not have nodules. In the comparison of prostate weight, presence/absence of nodules and the Gleason score (dichotomized or not), significant differences were found between the group with hypervascularization and the group with normal vascularization (Table 1).

In the univariate analysis, hypervascularization and PSA $\geq 20 \mathrm{ng} / \mathrm{ml}$ were individually associated with a poorer prognosis (Table 2).

\begin{tabular}{|c|c|c|c|}
\hline Feature & OR & $\mathbf{9 5 \%}$ CI & p-value* \\
\hline Hypervascularization & 4.1 & $2.598-6.554$ & $<0.001$ \\
\hline PSA $\geq 20 \mathrm{ng} / \mathrm{ml}$ & 5.3 & $2.335-12.086$ & $<0.001$ \\
\hline Age $>65$ y.o. & 0.9 & $0.525-1.544$ & 0.738 \\
\hline
\end{tabular}

Table 2: Univariate analysis.

$\mathrm{OR}=$ odds ratio; $\mathrm{CI}=$ confidence interval.

*Multivariate analysis.

These two variables remained independent prognostic factors in the multivariate analysis (Table 3).

\begin{tabular}{|c|c|c|c|}
\hline Feature & OR & $\mathbf{9 5 \%}$ CI & p-value* \\
\hline Hypervascularization & 7.4 & $2.841-19.449$ & $<0.001$ \\
\hline PSA $\geq 20 \mathrm{ng} / \mathrm{ml}$ & 4.7 & $1.928-11.483$ & 0.001 \\
\hline
\end{tabular}

Table 3: Multivariate analysis (Binary Logistic Regression).

\section{Discussion}

The present study demonstrated that an increase in vascularization in cases of prostate cancer is associated with a worse Gleason score and, indirectly, a worse prognosis. Khanduri, et al. (2017), previously reported a study involving color Doppler flowmetry in the detection of prostate malignancy. It was found that moderate vascularization and focal vascular asymmetry were significantly associated with malignancy. ${ }^{16}$ In the multivariate analysis, the group with hypervascularization had a 7.4-fold greater chance of having a Gleason score $>7$. Moreover, patients with a Gleason score $>7$ had a 4.1-fold greater chance of exhibiting hypervascularization. Malignant areas of the prostate tend to have more vessels per $\mathrm{mm}^{2}$ than benign fragments and, consequently, greater blood flow evidenced by color Doppler ultrasound. This finding tends to direct biopsies to regions related to a higher Gleason score [25].

The diagnosis of prostate cancer is currently performed using clinical and laboratory exams, such as the digital rectal exam, the serum concentration of PSA and a histopathological analysis of an ultrasound-guided biopsy fragment graded using the Gleason score that is based on the degree of glandular differentiation and, consequently, tumor aggressivity [26]. Thus, the Gleason score has considerable prognostic power in cases of prostate cancer and continues to be an important factor to consider in the decision-making process for the most indicated form of treatment [27]. In a study, prognostic groups were formed considering both the probability of recurrence in the next five years and the Gleason score determined during the histopathological exam. The prognostic groups were classified from 1 to 5 . Group 1 corresponded to Gleason score $\leq 6$ in patients with well-formed glands and a $96 \%$ probability of being free of reoccurrence in the five years following prostatic resection, whereas prognostic Group 5 (Gleason score of 9 or 10) has a $26 \%$ probability of being free of recurrence in five years [28]. Then, inverse and significant correlation was demonstrated between increasing group grading and recurrence-free progression following prostatectomy or radiotherapy. Some studies disagreed with the use of transrectal ultrasound with a color Doppler, demonstrating the absence of benefit $[29,30]$. But the detection of abnormal blood flow patterns within prostatic tumors is the main application of Doppler ultrasound in prostate cancer imaging. In addition, color Doppler is an alternative to more-invasive procedures which can show an increased vascularization from hypo echoic nodules which is similar to benign hyperplastic nodules. Few cases of prostatic lesion had abnormal flow on color Doppler scanning which was no obviously identifiable abnormality on ultrasound scanning [31]. Our study revealed an association between hyper vascularization and a worse prognosis of prostate cancer based on the Gleason score. However, studies involving the analysis of tumoral vascularization, survival, quality of life and/or cure rates should be conducted to confirm this inference.

Like other tumors, increased angiogenesis is found in prostate cancer, resulting in an increase in the density of vessels [16]. Tumoral blood vessels also have random paths and increased tortuosity that can assist in the detection of tumors or the guiding of the biopsy using a color Doppler exam [16-25]. Louvar, et al. Evaluated prostate cancer biopsies using Doppler ultrasound and found no significant differences in vasculature variables between high-flow and normal-flow color Doppler findings but biopsies with highflow color Doppler had a mean Gleason score of 6.7, whereas those with normal-flow color Doppler had a mean score of $5.9(\mathrm{p}<0.025)[25]$.

PSA is routinely one of the first tests performed in men with symptoms that may be caused by prostate cancer. The concentration of this marker is directly related to the presence of prostate cancer, with an increase in PSA increasing the chance of having cancer [32]. It has prognostic power and enables the evaluation of the staging of the tumor, which exerts an influence on the treatment options as well as the 


\section{Clinical Pathology \& Research Journal}

monitoring of prostate cancer during and after treatment. The results of the present investigation and previous studies demonstrate an association between increased PSA and more aggressive tumors as well as an association with a higher Gleason score and a greater risk of recurrence $[32,33]$.

The use of color (Power) Doppler assesses vascular permeability, providing more hemodynamic information. However, the detection of hyper vascularized tumors may not increase because angiogenesis of most small tumors is below the flow range of the Doppler. Despite the promising findings, questions remain regarding the efficiency of color Doppler ultrasound due to the resolution of the images. Some researchers strongly defend the systematic use of this technology during guided biopsies due to the ability to diagnose advanced tumors [34]. High-resolution color Doppler ultrasound and tissue harmonic imaging have improved the capacity for detecting cancer [34]. Moreover, a biopsy guided to the tumor together with a biopsy of the potential pathway of tumor escape (such as a neurovascular bundle or nearby seminal vesicles) improves the determination of the staging of the cancer and the Gleason classification [34]. With these advantages, color Doppler ultrasound gained popularity over conventional transrectal ultrasound used as a diagnostic modality, as Doppler ultrasound offers benefits in the differentiation of cancer among other prostatic lesions [34]. While Doppler imaging may be effective in detecting increased blood density, it is only possible if is in larger macro vessels that may be found in late-stage higher Gleason-grade tumors [35]. In this study, we qualitatively evaluated the hyper vascularization (yes or no) and Gleason score. And, considering our findings, in the next time, a quantitative analysis of the hyper vascularization can also predict whether it is a moderately differentiated carcinoma or poorly differentiated carcinoma, clinically.

The diagnosis and treatment of prostate cancer remain challenging. Current screening methods include the determination of serum PSA levels, the digital rectal exam and transrectal ultrasound. Color Doppler ultrasound has better diagnostic and prognostic value due to its capacity to visualize vascular changes [16] but is not sufficient to replace systematic biopsy [17].

This study showed an association between tumoral hyper vascularization and higher Gleason scores (more aggressive tumors). Tumors with a Gleason score $>7$ had a 4.1fold greater chance of having a Doppler ultrasound finding of hyper vascularization. Moreover, hyper vascularized tumors had a 7.4-fold greater chance of having a Gleason score $>7$ in the histopathological analysis. These findings suggest an indirect association between hyper vascularization and a poorer prognosis, considering the previously described association between the Gleason score and prognosis of patients with prostate cancer. Thus, the use of Doppler ultrasound for prostate analysis can assist in the location of prostatic tumors in more advanced stages.

Funding: This work was supported by the Coordination for the Improvement of Higher Education Personnel (CAPES)/ Brazil.

\section{Conflicts of Interest/Competing Interests: None}

Acknowledgements: We thank Radiology Service of Santa Casa Hospital, Belo Horizonte, for providing technical support on the images acquisition.

\section{References}

1. Torre LA, Bray F, Siegel RL, Ferlay J, Tieulent JL, et al. (2015) Global cancer statistics, 2012. CA Cancer J Clin 65(2): 87-108.

2. (2015) Instituto Nacional do Câncer. Brasil: Estimativa 2016: incidência de câncer no Brasil.

3. Heidenreich A, Bastian PJ, Bellmunt J, Bolla M, Joniauet $S$, et al. (2014) EAU guidelines on prostate cancer. Part 1: screening, diagnosis, and local treatment with curative intent-update 2013. Eur Urol 65(1): 124-137.

4. Porzycki P, Ciszkowicz E (2019) Detection of individual prostate cancer via multiparametric magnetic resonance imaging in own material - initial experience. J Contemp Brachytherapy 11(6): 541-546.

5. Girometti R, Cereser L, Bonato F (2019) Evolution of prostate MRI: from multiparametric standard to less-isbetter and different-is better strategies. Eur Radiol Exp 3(1): 5 .

6. Byar DP, Mostofi FK (1972) Carcinoma of the prostate: prognostic evaluation of certain pathologic features in 208 radical prostatectomies. Examined by the stepsection technique. Cancer 30(1):5-13.

7. Nishida N, Yano H, Nishida T, Kamura T, Kojiro M (2006) Angiogenesis in cancer. Vasc Health Risk Manag 2(3): 213-219.

8. Folkman J, Watson K, Ingber D (1989) Induction of angiogenesis during the transition from hyperplasia to neoplasia. Nature 339(6219): 58-61.

9. Ferrara KW, Merritt CRB, Burns PN, Foster FS, Mattrey RF, et al. (2000) Evaluation of tumor angiogenesis with US: Imaging, Doppler, and contrast agents. Academic Radiology 7(10): 824-839.

10. Kajita T, Ohta Y, Kimura K, Tamura M, Tanaka Y, et al. 


\section{Clinical Pathology \& Research Journal}

(2001) The expression of vascular endothelial growth factor $\mathrm{C}$ and its receptors in non-small cell lung cancer. Br J Cancer 85(2): 255-260.

11. Nishida N, Yano H, Komai K, Nishida T, Kamura T, et al. (2004) Vascular endothelial growth factor $C$ and vascular endothelial growth factor receptor 2 are related closely to the prognosis of ovarian carcinoma. Cancer 101(6): 1364-1374.

12. Amioka T, Kitadai Y, Tanaka S, Haruma K, Yoshihara $M$, et al. (2002) Vascular endothelial growth factor-C expression predicts lymph node metastasis of human gastric carcinomas invading the submucosa. Eur J Cancer 38(10): 1413-1419.

13. Charoenrat PO, Rhys Evans P, Eccles SA (2001) Expression of vascular endothelial growth factor family members in head and neck squamous cell carcinoma correlates with lymph node metastasis. Cancer 92(3): 556-568.

14. Weidner N, Semple JP, Welch WR, Folkman J (1991) Tumor angiogenesis and metastasis--correlation in invasive breast carcinoma. N Engl J Med 324(1): 1-8.

15. Bigler SA, Deering RE, Brawer MKM (1993) Comparison of microscopic vascularity in benign and malignant prostate tissue. Hum Pathol 24(2): 220-226.

16. Khanduri S, Katyal G, Goyal A, Bhagat S, Yadav S, et al. (2017) Evaluation of Prostatic Lesions by Transrectal Ultrasound, Color Doppler, and the Histopathological Correlation. Cureus 9(7): 1422.

17. Halpern EJ (2006) Contrast-Enhanced Ultrasound Imaging of Prostate Cancer. Rev Urol 8(1): 29-37.

18. Bjartell A (2006) Words of wisdom. The 2005 International Society of Urological Pathology (ISUP) Consensus Conference on Gleason Grading of Prostatic Carcinoma. Eur Urol 49(4): 758-759.

19. Buyyounouski MK, Choyke PL, McKenney JK, Sartor O, Sandler HM, et al. (2017) Prostate cancer - major changes in the American Joint Committee on Cancer eighth edition cancer staging manual. CA Cancer J Clin 67(3): 245-253.

20. Allan PL, Dubbins PA, Pozniak, MA, McDicken WN (2006) Ultra-sonografia com Doppler na Prática Clínica, $1^{\text {st }}(\mathrm{edn})$, Rio de Janeiro: Elsevier.

21. Epstein JI, Allsbrook WC, Amin MB, Egevad LL (2005) The 2005 International Society of Urological Pathology (ISUP) Consensus Conference on Gleason Grading of Prostatic Carcinoma. Am J Surg Pathol 29(9): 1228-1242.

22. Gerstenbluth RE, Seftel AD, Hampel N, Oefelein MG, Resnick MI (2002) The accuracy of the increased prostate specific antigen level (greater than or equal to $20 \mathrm{ng} . / \mathrm{ml}$.) in predicting prostate cancer: is biopsy always required? J Urol 168(5): 1990-1993.

23. da Cruz JAS, Passerotti CC, Dos Reis ST, Guariero MES, de Campos OD, et al. (2017) Is age an independent factor for prostate cancer? A paired analysis. Curr Urol 9(4): 183-187.

24. American Cancer Society (2017) Tests for Prostate Cancer, pp: 1-11.

25. Louvar E, Littrup PJ, Goldstein A, Yu L, Sakr W, et al. (1998) Correlation of color Doppler flow in the prostate with tissue microvascularity. Cancer 83(1): 135-140.

26. Gunderson LL, Tepper JE (2012) Clinical radiation oncology, $4^{\text {th }}(\mathrm{edn})$, Philadelphia, Elsevier, pp: 1648.

27. Muhammad A (2019) Prognostic Adenocarcinoma: A Grading from Gleason to the New Grade-Group System: A Historical and Critical Review. APJCC 20(3): 661-666.

28. Epstein JI, Zelefsky MJ, Sjoberg DD, Nelson JB, Egevad L, et al. (2016) A contemporary prostate cancer grading system: A validated alternative to the Gleason score. Eur Urol 69(3): 428-435.

29. Halpern EJ, Strup SE (2000) Using gray scale, color and power Doppler sonography to detect prostatic cancer. AJR Am J Roentgenol 74(3): 623-627.

30. Halpern EJ, Frauscher F, Strup SE, Nazarian LN, Kane PO, et al. (2002) Prostate: high-frequency Doppler US imaging for cancer detection. Radiology 225(1): 71-77.

31. Rifkin MD, Sudakoff GS, Alexander AA (1993) Prostate: Techniques,results, and potential applications of color Doppler US scanning. Radiology 186(2): 509-513.

32. Cheng S, Rifkin MD (2001) Color doppler imaging of the prostate: important adjunct to end orectal ultrasound of the prostate in the diagnosis of prostate cancer. Ultrasound Q 17(3): 185-189.

33. Huang ST, Hsieh ML (2008) Evaluation of resistance index in patients with prostate cancer. Anticancer Res 28(4A): 1985-1988.

34. Lee F, Bahn DK, Siders DB, Greene C (1998) The role of TRUS-guided biopsies for determination of internal and external spread of prostate cancer. Semin Urol Oncol 16(3): 129-136.

35. Sarkar S, Das S (2016) A Review of Imaging Methods for Prostate Cancer Detection. Biomed Eng Comput Biol $7(1): 1-15$.

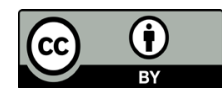

